\title{
THE RATIONALITY PROBLEM FOR NORM ONE TORI
}

\author{
SHIZUO ENDO
}

\section{To the memory of Professor Masayoshi Nagata}

\begin{abstract}
We consider the problem of whether the norm one torus defined by a finite separable field extension $K / k$ is stably (or retract) rational over $k$. This has already been solved for the case where $K / k$ is a Galois extension. In this paper, we solve the problem for the case where $K / k$ is a non-Galois extension such that the Galois group of the Galois closure of $K / k$ is nilpotent or metacyclic.
\end{abstract}

\section{Introduction}

Let $K / k$ be a finite separable field extension, and denote by $R_{K / k}^{(1)}\left(\mathbb{G}_{m}\right)$ the norm one torus defined by $K / k$, as usual (see, e.g., [V]).

The purpose of this paper is to determine whether the torus $T=$ $R_{K / k}^{(1)}\left(\mathbb{G}_{m}\right)$ is stably (or retract) rational over $k$. For the case where $K / k$ is Galois, this problem was solved completely in [EM2] and [S]. Hence, we have only to consider this for the case where $K / k$ is non-Galois.

Assume that $K / k$ is non-Galois, and let $L / k$ be the Galois closure of $K / k$. Let $G=\operatorname{Gal}(L / k)$, and let $H=\operatorname{Gal}(L / K)$. The main results in this paper are the following.

[I] Assume that $G$ is a nilpotent group. Then $T$ is not retract rational over $k$.

[II] Assume that $G$ is a metacyclic group. Then $T$ is always retract rational over $k$, and the following conditions are equivalent:

(1) $T$ is stably rational over $k$;

(2) $G$ is the dihedral group $D_{n}$ of order $2 n$ with $n$ odd or the direct product of the cyclic group $C_{m}$ of order $m$ and the dihedral group $D_{n}$ of order $2 n$, where $m, n$ are odd, $m, n \geqq 3,(m, n)=1$, and $H \subseteq D_{n}$ is of order 2 .

Received December 22, 2009. Accepted September 13, 2010.

2010 Mathematics Subject Classification. 14E08, 20C10. 
[III] Assume that $G=A_{n}, n \geqq 3$, the alternating group on $n$ letters, and that $H=A_{n-1} \subseteq G$, where $H$ is the stabilizer of one of the letters in G. Then,

(1) $T$ is retract rational over $k$ if and only if $n$ is a prime;

(2) for some $t \geqq 1, T^{(t)}$, the product of $t$ copies of $T$, is stably rational over $k$ if and only if $n=3,5$.

For the case of $G$ metacyclic, it is an immediate consequence of [EM2, (1.5)] and $[\mathrm{S},(3.14)]$ that $T$ is retract rational over $k$. It should be noted that partial results of [I] and [II] have already been given in [CS1].

[I] and [II] are final answers to the problem for the cases of nilpotent groups and metacyclic groups, respectively. [III] can be regarded as an additional remark on the result for symmetric groups in [CS2], [1B], [CK], [LL], and so forth. We will also give another proof of the result for symmetric groups.

\section{$\S 1$. Preliminaries}

Let $G$ be a finite group. A $G$-module means a finitely generated left $G$ module, and a $G$-module with a $\mathbb{Z}$-basis is said to be a $G$-lattice. A $G$-lattice $M$ is said to be a permutation $G$-lattice if it has a $\mathbb{Z}$-basis permuted by $G$, that is, if $M \cong \bigoplus_{1 \leqq i \leqq m} \mathbb{Z} G / H_{i}$ for subgroups $H_{1}, H_{2}, \ldots, H_{m} . M$ is said to be invertible if it is a direct summand of a permutation $G$-lattice. $M$ is said to be a quasi-permutation if there exists an exact sequence of $G$-lattices

$$
0 \rightarrow M \rightarrow U \rightarrow V \rightarrow 0
$$

where $U$ and $V$ are permutation lattices. $M$ is said to be quasi-invertible if it is a direct summand of a quasi-permutation $G$-lattice. The dual lattice $\operatorname{Hom}_{\mathbb{Z}}(M, \mathbb{Z})$ of a $G$-lattice $M$ is denoted by $M^{\circ}$.

For a subgroup $H$ of $G$, there exists an exact sequence of $G$-lattices

$$
0 \rightarrow I_{G / H} \rightarrow \mathbb{Z} G / H \stackrel{\varepsilon}{\rightarrow} \mathbb{Z} \rightarrow 0
$$

where $\varepsilon$ is the augmentation map and $I_{G / H}=\operatorname{Ker} \varepsilon$. The dual lattice $J_{G / H}=$ $\left(I_{G / H}\right)^{\circ}$ of $I_{G / H}$ will play a central part in this paper.

When $I_{G / H}$ and $J_{G / H}$ are examined, $H$ can be assumed to contain no normal subgroup of $G$ except $\{1\}$. In fact, let $N \subseteq H$ be a maximal subgroup which is normal in $G$, set $\bar{G}=G / N$, and set $\bar{H}=H / N$. Then $I_{\bar{G} / \bar{H}}=I_{G / H}$ and $J_{\bar{G} / \bar{H}}=J_{G / H}$, and therefore we may use $\bar{G}$ and $\bar{H} \operatorname{instead}$ of $G$ and $H$, where $\bar{H}$ contains no normal subgroup of $\bar{G}$ except $\{1\}$. 
Throughout this paper, a finite group is said to be a metacyclic group if all its Sylow subgroups are cyclic.

Let $k$ be a field, let $L$ be a finite Galois extension of $k$, and let $G=$ $\operatorname{Gal}(L / k)$. Let $M$ be a $G$-lattice with a $\mathbb{Z}$-basis $\left\{u_{1}, u_{2}, \ldots, u_{n}\right\}$. Define the action of $G$ on the rational function field $L\left(X_{1}, X_{2}, \ldots, X_{n}\right)$ with variables $X_{1}, X_{2}, \ldots, X_{n}$ over $L$, as an extension of the action of $G$ over $L$, as follows. For each $\sigma \in G$,

$$
\sigma\left(X_{i}\right)=\prod_{j=1}^{n} X_{j}^{m_{i j}}, \quad 1 \leqq i \leqq n,
$$

when $\sigma u_{i}=\sum_{j=1}^{n} m_{i j} u_{j}, m_{i j} \in \mathbb{Z}$, and denote $L\left(X_{1}, X_{2}, \ldots, X_{n}\right)$ with this action of $G$ by $L(M)$.

For a given $G$-lattice $M$, there exists an algebraic torus $T$ defined over $k$ and split over $L$ such that the character group of $T$ is isomorphic to $M$ as $G$-lattices, and the invariant subfield $L(M)^{G}$ of $L(M)$ can be identified with the function field of $T$.

An extension field $F$ of a basic field $k$ is said to be rational over $k$ if it is generated over $k$ by a finite number of elements of $F$ which are algebraically independent over $k . F$ is said to be stably rational over $k$ if there exists an extension field of $F$ which is rational over each of $k$ and $F$. Further, $F$ is said to be retract rational over $k$ if there exists an extension field $k\left(x_{1}, x_{2}, \ldots, x_{n}\right)$ of $F$ rational over $k$ where $x_{1}, x_{2}, \ldots, x_{n}$ are algebraically independent over $k$, and if $F$ is the quotient field of a $k$-subalgebra $A$ of $F$ such that, for some nonzero element $s$ of $k\left[x_{1}, x_{2}, \ldots, x_{n}\right]$, we have $A \subseteq k\left[x_{1}, x_{2}, \ldots, x_{n}\right][1 / s]$ and a $k$-algebra homomorphism

$$
\theta: k\left[x_{1}, x_{2}, \ldots, x_{n}\right][1 / s] \rightarrow A
$$

whose restriction to $A$ is the identity on $A$. More generally, $F$ is said to be unirational over $k$ if there exists an extension field of $F$ which is rational over $k$.

It is easy to see that

$$
\text { rational } \Longrightarrow \text { stably rational } \Longrightarrow \text { retract rational } \Longrightarrow \text { unirational. }
$$

It should be noted that every algebraic torus defined by a separable extension of a field $k$ is unirational over $k$.

We now have the following.

THEOREM 1.1. Let $L / k$ be a finite Galois field extension with a group $G$, and let $M$ be a $G$-lattice. Then, 
(1) $M$ is a quasi-permutation $G$-lattice if and only if $L(M)^{G}$ is stably rational over $k$ (see, e.g., [EM1, (1.6)]);

(2) $M$ is a quasi-invertible $G$-lattice if and only if $L(M)^{G}$ is retract rational over $k$ (see $[S,(3.14)])$.

Let $k$ be a field, and let $K / k$ be a finite separable extension. Let $L / k$ be the Galois closure of $K / k$, let $G=\operatorname{Gal}(L / k)$, and let $H=\operatorname{Gal}(L / K) \subseteq G$. The norm one torus $R_{K / k}^{(1)}\left(\mathbb{G}_{m}\right)$ defined by $K / k$ has the lattice $J_{G / H}$ as its character lattice and the field $L\left(J_{G / H}\right)^{G}$ as its function field (see [V]). Note that $H$ contains no normal subgroup of $G$ except $\{1\}$, since $L / k$ is the Galois closure of $K / k$. For the case where $K / k$ is Galois (i.e., $H=\{1\}$ ), the $G$-lattices $I_{G / H}$ and $J_{G / H}$ are denoted by $I_{G}$ and $J_{G}$, respectively.

For the case where $K / k$ is Galois, we have the following.

THEOREM 1.2. Let $K / k$ be a finite Galois field extension with a group $G$. Then,

(1) $R_{K / k}^{(1)}\left(\mathbb{G}_{m}\right)$ is retract rational over $k$ if and only if $G$ is metacyclic (see [EM2, (1.5)], [S, (3.14)]);

(2) $R_{K / k}^{(1)}\left(\mathbb{G}_{m}\right)$ is stably rational over $k$ if and only if $G$ is a cyclic group, or a direct product of a cyclic group of order $m$ and a group $\langle\sigma, \tau| \sigma^{n}=$ $\left.\tau^{2^{d}}=1, \tau \sigma \tau^{-1}=\sigma^{-1}\right\rangle$, where $d, m \geqq 1, n \geqq 3, m, n$ odd, and $(m, n)=1$ (see [EM2, (2.3)]).

Therefore, in this paper, we will consider only the case where $K / k$ is non-Galois, that is, the case where $H \neq\{1\}$.

Let $G$ be a finite group. Let $H_{1}, H_{2}, \ldots, H_{t}, t \geqq 2$ be subgroups of $G$, and let $\varepsilon_{i}: \mathbb{Z} G / H_{i} \rightarrow \mathbb{Z}, 1 \leqq i \leqq t$, be the augmentation maps. Then the multiaugmentation map

$$
\varepsilon=\left(\varepsilon_{1}, \varepsilon_{2}, \ldots, \varepsilon_{t}\right): \mathbb{Z} G / H_{1} \oplus \mathbb{Z} G / H_{2} \oplus \cdots \oplus \mathbb{Z} G / H_{t} \rightarrow \mathbb{Z}
$$

is defined by sending $u=\left(u_{i}\right) \in \bigoplus_{i=1}^{t} \mathbb{Z} G / H_{i}$ to $\sum_{i=1}^{t} \varepsilon_{i}\left(u_{i}\right) \in \mathbb{Z}$.

The following proposition on multiaugmentation maps is simple but very useful.

Proposition 1.3. Let $G$ be a finite group, and let $H_{1}, H_{2}, \ldots, H_{t}, t \geqq 2$ be subgroups of $G$ such that $H_{t-1} \supseteq H_{t}$. Let $\varepsilon_{i}: \mathbb{Z} G / H_{i} \rightarrow \mathbb{Z}, 1 \leqq i \leqq t$, be the augmentation maps. Further, let 
$\varepsilon=\left(\varepsilon_{1}, \varepsilon_{2}, \ldots, \varepsilon_{t-1}, \varepsilon_{t}\right): \mathbb{Z} G / H_{1} \oplus \mathbb{Z} G / H_{2} \oplus \cdots \oplus \mathbb{Z} G / H_{t-1} \oplus \mathbb{Z} G / H_{t} \rightarrow \mathbb{Z}$,

$$
\varepsilon^{\prime}=\left(\varepsilon_{1}, \varepsilon_{2}, \ldots, \varepsilon_{t-1}\right): \mathbb{Z} G / H_{1} \oplus \mathbb{Z} G / H_{2} \oplus \cdots \oplus \mathbb{Z} G / H_{t-1} \rightarrow \mathbb{Z},
$$

be the multiaugmentation maps, set $I=\operatorname{Ker} \varepsilon, I^{\prime}=\operatorname{Ker} \varepsilon^{\prime}$, and set $J=I^{\circ}$, $J^{\prime}=\left(I^{\prime}\right)^{\circ}$. Then $I \cong I^{\prime} \oplus \mathbb{Z} G / H_{t}$ and $J \cong J^{\prime} \oplus \mathbb{Z} G / H_{t}$.

Proof. Define $\delta_{t}: \mathbb{Z} G / H_{t} \rightarrow \mathbb{Z} G / H_{t-1}$ by $\rho H_{t} \rightarrow \rho H_{t-1}, \rho \in G$, and define

$$
\begin{aligned}
\delta= & \left(1,1, \ldots, 1, \delta_{t}\right): \mathbb{Z} G / H_{1} \oplus \mathbb{Z} G / H_{2} \oplus \cdots \oplus \mathbb{Z} G / H_{t-1} \oplus \mathbb{Z} G / H_{t} \\
& \rightarrow \mathbb{Z} G / H_{1} \oplus \mathbb{Z} G / H_{2} \oplus \cdots \oplus \mathbb{Z} G / H_{t-1}
\end{aligned}
$$

by sending $\left(u_{1}, u_{2}, \ldots, u_{t-1}, u_{t}\right)$ to $\left(u_{1}, u_{2}, \ldots, u_{t-1}+\delta_{t}\left(u_{t}\right)\right)$. Then $\delta$ is a split surjection and $\operatorname{Ker} \delta\left(\cong \mathbb{Z} G / H_{t}\right) \subseteq I$. Hence, we can form the following commutative diagram with exact rows and columns:

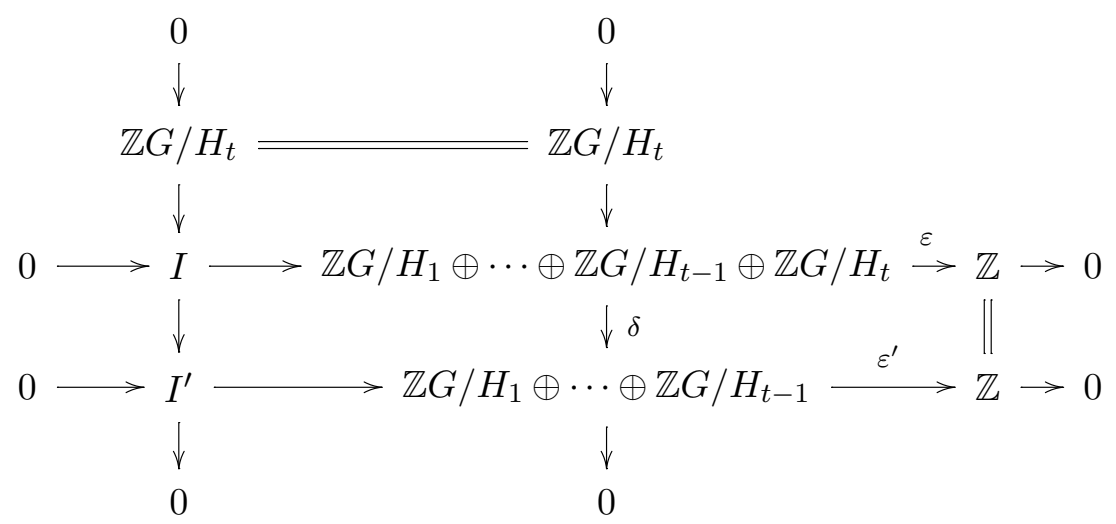

Then the first column is also split, and so $I \cong I^{\prime} \oplus \mathbb{Z} G / H_{t}$ and $J \cong J^{\prime} \oplus$ $\mathbb{Z} G / H_{t}$.

Corollary 1.4. Let $G$ be a finite group, and let

$$
\varepsilon^{(t)}:[\mathbb{Z} G]^{(t)} \rightarrow \mathbb{Z}, \quad t \geqq 2,
$$

be the multiaugmentation map of $[\mathbb{Z} G]^{(t)}$, the direct sum of $t$ copies of $\mathbb{Z} G$, on $\mathbb{Z}$ defined as in Proposition 1.3 by augmentation map $: \mathbb{Z} G \rightarrow \mathbb{Z}$. Let $I=\operatorname{Ker} \varepsilon^{(t)}$, and let $J=I^{\circ}$. Then $I \cong I_{G} \oplus[\mathbb{Z} G]^{(t-1)}$, and hence $J \cong J_{G} \oplus$ $[\mathbb{Z} G]^{(t-1)}$.

Note that special cases of Proposition 1.3 and Corollary 1.4 have been used in $[\mathrm{E}]$ and $[\mathrm{CK}]$. 
A lattice $M$ over a finite group $G$ is said to be coflasque if $H^{1}\left(G^{\prime}, M\right)=0$ for any subgroup $G^{\prime}$ of $G$. Every invertible lattice is coflasque. For any $G$-lattice $M$, we can construct an exact sequence

$$
0 \rightarrow N \rightarrow U \rightarrow M \rightarrow 0
$$

where $U$ is permutation and $N$ is coflasque (see [EM2, (1.1)]). This is said to be a coflasque resolution of $M$.

Proposition 1.5. Let $G$ be a finite group, and let $0 \rightarrow N \rightarrow U \rightarrow M \rightarrow 0$ be an exact sequence of $G$-lattices with $U$ permutation. Then,

(1) $M^{\circ}$ is a quasi-permutation if and only if $N$ is a quasi-permutation;

(2) $M^{\circ}$ is quasi-invertible if and only if $N$ is quasi-invertible.

Suppose further that $N$ is coflasque. Then,

(3) $M^{\circ}$ is quasi-invertible if and only if $N$ is invertible.

Proof. For example, see the proof of [EM2, (1.6)].

Corollary 1.6. A lattice over a finite group $G$ is quasi-invertible if and only if it is quasi-invertible over every Sylow subgroup of $G$.

Proof. It is well known (see, e.g., [EM2, (1.4)]) that a $G$-lattice is invertible if and only if it is invertible over every Sylow subgroup of $G$. Therefore, the assertion follows directly from Proposition 1.5.

The following proposition is only a slight generalization of Theorem 1.2(1), but this is useful for our problem.

Proposition 1.7. Let $G$ be a finite group, and let $H$ be a nonnormal Hall subgroup of $G$. Then $J_{G / H}$ is quasi-invertible over $G$ if and only if all Sylow p-subgroups of $G$ are cyclic for any prime $p \mid[G: H]$.

Proof. Suppose that there exists a noncyclic Sylow $p$-subgroup $P$ of $G$ for some prime $p \mid[G: H]$. Then $\mathbb{Z} G / H$ is $\mathbb{Z} P$ free, and therefore $\mathbb{Z} G / H \cong$ $[\mathbb{Z} P]^{(t)}, t \geqq 1$. Hence, by Corollary $1.4, J_{G / H} \cong J_{P} \oplus[\mathbb{Z} P]^{(t-1)}$. However, since $P$ is noncyclic, $J_{P}$ is not quasi-invertible over $P$. Thus, $J_{G / H}$ is not quasi-invertible over $G$.

On the other hand, suppose that Sylow $p$-subgroups of $G$ are cyclic for any prime $p \mid[G: H]$. Let $p$ be a prime divisor of $|G|$, and let $P$ be a Sylow $p$ subgroup. Assume first that $p \mid[G: H]$. Then, as above, $\mathbb{Z} G / H \cong[\mathbb{Z} P]^{(t)}, t \geqq$ 1 , as $P$-lattices, and hence, by Corollary $1.4, J_{G / H} \cong J_{P} \oplus[\mathbb{Z} P]^{(t-1)}$. Since $P$ is cyclic by assumption, $J_{P}$ is quasi-invertible. This shows that $J_{G / H}$ is 
quasi-invertible over $P$. Next, assume that $p|| H \mid$. As $H$ is a Hall subgroup, we have $p \nmid[G: H]$, and then the action of $P$ on $G / H$ has a fixed point. Therefore, $J_{G / H}$, as a $P$-lattice, is a direct summand of $\mathbb{Z} G / H$, which shows also that $J_{G / H}$ is invertible over $P$. Hence, in both cases, $J_{G / H}$ is quasiinvertible over $P$. Thus, it follows from Corollary 1.6 that $J_{G / H}$ is quasiinvertible over $G$.

\section{$\S 2 . \quad$ Nilpotent groups}

In this section, we will prove the following.

THEOREM 2.1. Let $K / k$ be a finite non-Galois, separable field extension, and let $L / k$ be the Galois closure of $K / k$. Assume that the Galois group of $L / k$ is nilpotent. Then the norm one torus $R_{K / k}^{(1)}\left(\mathbb{G}_{m}\right)$ defined by $K / k$ is not retract rational over $k$.

Let $G=\operatorname{Gal}(L / k)$, and let $H=\operatorname{Gal}(L / K) \subseteq G$. In order to prove Theorem 2.1, it suffices to show by Theorem $1.1(2)$ that the $G$-lattice $J=J_{G / H}$ is not quasi-invertible.

We can reduce Theorem 2.1 to the case where $G$ is a $p$-group for a prime $p$. In fact, given a nilpotent group $G$ and a nonnormal subgroup $H \subseteq G$, there exists a Sylow $p$-subgroup $P$ for some $p|| G \mid$ such that $P^{\prime}=P \cap$ $H$ is nonnormal in $P$, because nilpotent groups $G$ and $H$ are expressible uniquely as the direct products of their Sylow subgroups. Then we have $\mathbb{Z} G / H \cong\left[\mathbb{Z} P / P^{\prime}\right]^{(t)}$ for some $t \geqq 1$ as $P$-lattices, and so, by Proposition 1.3, $J_{G / H} \cong J_{P / P^{\prime}} \oplus\left[\mathbb{Z} P / P^{\prime}\right]^{(t-1)}$ as $P$-lattices. Accordingly, it follows that $J_{G / H}$ is not quasi-invertible over $G$ when $J_{P / P^{\prime}}$ is not quasi-invertible over $P$.

From now on, we assume that $G$ is a $p$-group and that $H \subseteq G$ contains no normal subgroup of $G$ except $\{1\}$.

We will prove step by step that $J_{G / H}$ is not quasi-invertible over $G$.

STEP 1. Case where the center of $\mathrm{G}$ is not cyclic.

Proof. Let $Z=Z(G)$ be the center of $G$. Since $H$ contains no normal subgroup of $G$ except $\{1\}$, we have $H \cap Z=\{1\}$, and so $\mathbb{Z} G / H \cong[\mathbb{Z} Z]^{(t)}$ for some $t \geqq 1$ as $Z$-lattices. Then, from Corollary 1.4 , it follows that $J_{G / H} \cong$ $J_{Z} \oplus[\mathbb{Z} Z]^{(t-1)}$. Since $Z$ is not cyclic by the assumption, $J_{Z}$ is not quasiinvertible over $Z$ by Theorem $1.2(1)$, and so $J_{G / H}$ is not quasi-invertible over $G$.

According to Step 1, we may assume from now that the center $Z(G)$ of $G$ is cyclic. 
Step 2. Case where $p$ is odd.

Proof. By [Be, (1.4)], there exists a normal subgroup of $G$ as follows:

$$
N=\left\langle\sigma, \tau \mid \sigma^{p}=\tau^{p}=1, \sigma \tau=\tau \sigma\right\rangle
$$

Then, by the above assumption, we may suppose that $N \cap Z(G)=\langle\sigma\rangle$.

Suppose first that $H \cap N=\{1\}$. Then we have $\mathbb{Z} G / H \cong[\mathbb{Z} N]^{(t)}$ for some $t \geqq 1$, as $N$-lattices, and therefore by Corollary $1.4, J_{G / H} \cong J_{N} \oplus[\mathbb{Z} N]^{(t-1)}$, as $N$-lattices. Since $N$ is not cyclic, $J_{N}$ is not quasi-invertible over $N$ by Theorem 1.2(1), and hence $J_{G / H}$ is also not quasi-invertible over $G$.

Next, suppose that $H \cap N \neq\{1\}$. Then we may assume that $H \cap N=\langle\tau\rangle$. As is easily seen, the subgroups $\langle\tau\rangle,\langle\tau \sigma\rangle,\left\langle\tau \sigma^{2}\right\rangle, \ldots,\left\langle\tau \sigma^{p-1}\right\rangle$ are conjugate under $G$, because $\langle\tau\rangle$ is not normal in $G$ and $\sigma \in Z(G)$, and so, as $N$-lattices,

$$
\mathbb{Z} G / H \cong\left[\mathbb{Z} N /\langle\tau\rangle \oplus \mathbb{Z} N /\langle\tau \sigma\rangle \oplus \cdots \oplus \mathbb{Z} N /\left\langle\tau \sigma^{p-1}\right\rangle\right]^{(t)}
$$

for some $t \geqq 1$. Let

$$
\varepsilon: \mathbb{Z} N /\langle\tau\rangle \oplus \mathbb{Z} N /\langle\tau \sigma\rangle \oplus \cdots \oplus \mathbb{Z} N /\left\langle\tau \sigma^{p-1}\right\rangle \rightarrow \mathbb{Z}
$$

be the multiaugmentation map, and set $J=[\operatorname{Ker} \varepsilon]^{\circ}$. Then it follows from Proposition 1.3 that

$$
J_{G / H} \cong J \oplus\left[\mathbb{Z} N /\langle\tau\rangle \oplus \mathbb{Z} N /\langle\tau \sigma\rangle \oplus \cdots \oplus \mathbb{Z} N /\left\langle\tau \sigma^{p-1}\right\rangle\right]^{(t-1)} .
$$

Since $J$ is not quasi-invertible over $N$ by [E, Theorem 2(2)], this implies that $J_{G / H}$ is not quasi-invertible over $G$.

The following 2-groups are said to be of maximal class (see [Be, p. 26, Definition 2 and (1.7)]):

- the dihedral group

$D_{2^{n}}=\left\langle\sigma, \tau \mid \sigma^{2^{n}}=\tau^{2}=1, \tau \sigma \tau^{-1}=\sigma^{-1}\right\rangle, n \geqq 2$,

- the generalized quaternion group

$Q_{2^{n}}=\left\langle\sigma, \tau \mid \sigma^{2^{n}}=1, \sigma^{2^{n-1}}=\tau^{2}, \tau \sigma \tau^{-1}=\sigma^{-1}\right\rangle, n \geqq 2$,

- the semidihedral group

$S D_{2^{n}}=\left\langle\sigma, \tau \mid \sigma^{2^{n}}=\tau^{2}=1, \tau \sigma \tau^{-1}=\sigma^{-1+2^{n-1}}\right\rangle, n \geqq 3$.

Any subgroup $\neq\{1\}$ of the group $Q_{2^{n}}$ contains the center $Z\left(Q_{2^{n}}\right)=$ $\left\langle\sigma^{2^{n-1}}\right\rangle$, and so $Q_{2^{n}}$ can be omitted from the object of our consideration.

SteP 3. Case where $p=2$ and $G$ is of maximal class. 
Proof. Assume that $G=D_{2^{n}}$. Then $H$ is one of the subgroups $\langle\tau\rangle,\langle\tau \sigma\rangle$, $\ldots,\left\langle\tau \sigma^{2^{n-1}}\right\rangle$, and therefore we may assume that $H=\langle\tau\rangle$. Define $N=\left\langle\sigma^{2}\right.$, $\tau \sigma\rangle$. Then $N$ is normal in $G$ and $N \cong D_{2^{n-1}}(n \geqq 3)$ or the elementary abelian group of order 4 . Further, we have $\mathbb{Z} G / H \cong \mathbb{Z} N$ as $N$-lattices, and hence $J_{G / H}=J_{N}$ is not quasi-invertible over $N$, again by Theorem 1.2(1). Thus, we conclude that $J_{G / H}$ is not quasi-invertible over $G$.

Next, assume that $G=S D_{2^{n}}$. Then $H$ is one of the subgroups $\langle\tau\rangle,\left\langle\tau \sigma^{2}\right\rangle$, $\ldots,\left\langle\tau \sigma^{2\left(2^{n-1}-1\right)}\right\rangle$, and therefore we may assume that $H=\langle\tau\rangle$. Note that the subgroups $\langle\tau \sigma\rangle,\left\langle\tau \sigma^{3}\right\rangle, \ldots,\left\langle\tau \sigma^{2^{n}-1}\right\rangle$ of $G$ contain the center $Z(G)=\left\langle\sigma^{2^{n-2}}\right\rangle$. Set $N=\left\langle\sigma^{2}, \tau \sigma\right\rangle$. Then $N$ is normal in $G$ and $N \cong Q_{2^{n-1}}(n \geqq 3)$. Further, we have $\mathbb{Z} G / H \cong \mathbb{Z} N$ as $N$-lattices, and hence, along the same lines as in the dihedral case, we can show that $J_{G / H}$ is not quasi-invertible over $G$.

STEP 4. Case where a 2-group $G$ is not of maximal class and does not have the elementary abelian group of order 8 as its normal subgroup.

Proof. Since $G$ is not of maximal class, there exists an elementary abelian normal subgroup $E$ of order 4 in $G$ by [Be, (1.4)]. The centralizer $C_{G}(E)$ of $E$ in $G$ is normal in $G$, and by [Be, (1.8)], we have $E \subsetneq C_{G}(E)$. Then there is $\rho \in C_{G}(E)-E$ such that the class $\bar{\rho}$ of $\rho$ in $G / E$ is contained in the center of $G / E$ and is of order 2. Then $N=\langle\rho, E\rangle$ is an abelian, noncyclic normal subgroup of order 8 in $G$. However, by the assumption, $N$ is not elementary abelian, and therefore it can be expressed as follows:

$$
N=\left\langle\sigma, \tau \mid \sigma^{4}=\tau^{2}=1, \sigma \tau=\tau \sigma\right\rangle
$$

Since the conjugacy class of $\sigma$ in $G$ is contained in $\left\{\sigma, \sigma^{3}, \sigma \tau, \sigma^{3} \tau\right\}$, the conjugacy class of $\sigma^{2}$ in $G$ is $\left\{\sigma^{2}\right\}$, and so we have $\sigma^{2} \in Z(G)$. However, by assumption, $Z(G)$ is cyclic. Accordingly, the elements $\tau$ and $\sigma^{2} \tau$ of order 2 in $N$ must be conjugate under $G$.

Assume first that $H \cap N=\{1\}$. Then $\mathbb{Z} G / H \cong[\mathbb{Z} N]^{(t)}, t \geqq 1$, as $N$ lattices, and therefore, by Corollary 1.4, $J_{G / H} \cong J_{N} \oplus[\mathbb{Z} N]^{(\overline{t-1})}$, as $N$ lattices. Since $N$ is not cyclic, we can conclude that $J_{G / H}$ is not quasiinvertible.

Next, assume that $H \cap N \neq\{1\}$. Because $H \cap Z(G)=\{1\}, \sigma^{2} \notin H$, and so $H \cap N=\{\tau\}$ or $\left\{\tau \sigma^{2}\right\}$. As noted above, $\tau$ and $\tau \sigma^{2}$ are conjugate under $G$. Hence, we have $\mathbb{Z} G / H \cong\left[\mathbb{Z} N /\langle\tau\rangle \oplus \mathbb{Z} N /\left\langle\tau \sigma^{2}\right\rangle\right]^{(s)}, s \geqq 1$, as $N$-lattices. Let

$$
\varepsilon=\left(\varepsilon_{1}, \varepsilon_{2}\right): U=\mathbb{Z} N /\langle\tau\rangle \oplus \mathbb{Z} N /\left\langle\tau \sigma^{2}\right\rangle \rightarrow \mathbb{Z}
$$


be the multiaugmentation map, and set $J=[\operatorname{Ker} \varepsilon]^{\circ}$. Then, by Proposition 1.3, $J_{G / H} \cong J \oplus\left[\mathbb{Z} N /\langle\tau\rangle \oplus \mathbb{Z} N /\left\langle\tau \sigma^{2}\right\rangle\right]^{(s-1)}$ as $N$-lattices. According to Lemma 2.2 given at the end of this section, $J$ is not quasi-invertible over $N$. Thus, $J_{G / H}$ is not quasi-invertible over $G$.

STEP 5. Case where the 2-group $G$ has the elementary abelian group $E$ of order 8 as a normal subgroup.

Proof. Let $E=\left\langle\rho, \sigma, \tau \mid \rho^{2}=\sigma^{2}=\tau^{2}=1, \rho \sigma=\sigma \rho, \sigma \tau=\tau \sigma, \tau \rho=\rho \tau\right\rangle$. Since $Z(G)$ is cyclic and $E \cap Z(G) \neq\{1\}$, we may assume that $E \cap Z(G)=$ $\langle\rho\rangle$. Since $H$ contains no normal subgroup of $G$ except $\{1\}$, we have $H \cap$ $Z(G)=\{1\}$, and hence $\rho$ is not contained in any subgroup conjugate to $H$.

First, suppose that $|H \cap E|=1$, that is, that $H \cap E=\{1\}$. Then we have $\mathbb{Z} G / H \cong[\mathbb{Z} E]^{(t)}$ for some $t \geqq 1$ as $E$-lattices, and so the proof is similar to the previous one.

Second, suppose that $|H \cap E|=2$. Then we may assume that $H \cap E=\langle\sigma\rangle$. Let $E_{0}=\langle\rho, \sigma\rangle$. If $E_{0}$ is normal in $G$, then $\{\sigma, \sigma \rho\}$ is a conjugacy class of $G$. Then the subgroup $E_{1}=\langle\rho, \tau\rangle$ does not contain any of $\sigma$ and $\sigma \rho$. Hence, we have $\mathbb{Z} G / H \cong\left[\mathbb{Z} E_{1}\right]^{(t)}$ for some $t \geqq 1$ as $E_{1}$-lattices. On the other hand, if $E_{0}$ is not normal in $G$, then one of the subgroups $\langle\rho, \tau\rangle$ and $\langle\rho, \sigma \tau\rangle$ is normal in $G$, and we denote it by $E_{1}$. Then $E_{1} \cap H=\{1\}$, and therefore we have $\mathbb{Z} G / H \cong\left[\mathbb{Z} E_{1}\right]^{(t)}$ for some $t \geqq 1$ as $E_{1}$-lattices. Thus, the proof is done in the same way as in the first case.

Finally, suppose that $|H \cap E|=4$. In this case, we may assume that $H \cap E=\langle\sigma, \tau\rangle$. Now, all the subgroups of order 4 in $E$ are expressible as follows:

$$
\begin{gathered}
\langle\sigma, \tau\rangle, \quad\langle\rho \sigma, \tau\rangle, \quad\langle\sigma, \rho \tau\rangle, \quad\langle\rho \sigma, \sigma \tau\rangle, \\
\langle\rho, \sigma\rangle, \quad\langle\rho, \tau\rangle, \quad\langle\rho, \sigma \tau\rangle .
\end{gathered}
$$

The groups in the second row are not conjugate to those in the first row under $G$, because $E \cap Z(G)=\langle\rho\rangle$.

We will now show that the groups in the first row of the above list are conjugate under $G$. Let $E_{1}=\langle\rho, \sigma \tau\rangle$, let $E_{2}=\langle\rho, \sigma\rangle$, and let $E_{3}=\langle\rho, \tau\rangle$. It is easy to see that at least one of $E_{1}, E_{2}$, and $E_{3}$ is normal in $G$, and so we may assume that $E_{1}$ is normal in $G$. Then the centralizer $C_{G}(\sigma \tau)$ of $\sigma \tau$ in $G$ is a maximal subgroup of $G$; that is, $\left[G: C_{G}(\sigma \tau)\right]=2$. Note that $E_{2}$ and $E_{3}$ are either both normal or both nonnormal in $G$.

We first consider the case where both $E_{2}$ and $E_{3}$ are normal in $G$. Then both $C_{G}(\sigma)$ and $C_{G}(\tau)$, the centralizers of $\sigma$ and $\tau$ in $G$, are maximal in 
$G$. These three maximal subgroups of $G$ are distinct. In fact, if $C_{G}(\sigma)=$ $C_{G}(\tau)$, for example, then $C_{G}(\sigma)=C_{G}(\tau)=C_{G}(\sigma \tau)$, since $C_{G}(\sigma) \cap C_{G}(\tau) \subseteq$ $C_{G}(\sigma \tau)$. Setting $C=C_{G}(\sigma)=C_{G}(\tau)=C_{G}(\sigma \tau)$ and taking $\mu \in G-C$, we have $\mu \sigma \mu^{-1}=\rho \sigma, \mu \tau \mu^{-1}=\rho \tau$, and $\mu \sigma \tau \mu^{-1}=\rho \sigma \tau$, because $E_{1}, E_{2}$, and $E_{3}$ are normal in $G$. From the equalities $\mu \sigma \mu^{-1}=\rho \sigma$ and $\mu \tau \mu^{-1}=\rho \tau$, it follows that $\left(\mu \sigma \mu^{-1}\right)\left(\mu \tau \mu^{-1}\right)=(\rho \sigma)(\rho \tau)=\sigma \tau$. This contradicts obviously the third equality $\mu \sigma \tau \mu^{-1}=\rho \sigma \tau$. Now, let $\mu \in C_{G}(\sigma)-C_{G}(\tau)$ and $\nu \in$ $C_{G}(\tau)-C_{G}(\sigma)$. Then we have

$$
\begin{aligned}
\mu \sigma \mu^{-1} & =\sigma, & \mu \tau \mu^{-1} & =\rho \tau, \\
\nu \sigma \nu^{-1} & =\rho \sigma, & \nu \tau \nu^{-1} & =\tau, \\
(\nu \mu) \sigma(\nu \mu)^{-1} & =\rho \sigma, & (\nu \mu) \tau(\nu \mu)^{-1} & =\rho \tau .
\end{aligned}
$$

This implies that the groups given in the first row are conjugate under $G$.

Second, we consider the case where both $E_{2}$ and $E_{3}$ are nonnormal in $G$. In this case, the set $\{\sigma, \rho \sigma, \tau, \rho \tau\}$ is a conjugacy class of $G$, because $[G$ : $\left.C_{G}(\sigma)\right]=\left[G: C_{G}(\tau)\right]=4$. If $C_{G}(\sigma)=C_{G}(\tau)$, then $C=C_{G}(\sigma)=C_{G}(\tau) \subsetneq$ $C_{G}(\sigma \tau) \subsetneq G$. Let $\mu \in G-C_{G}(\sigma \tau)$. Since $\mu \notin C$, we have

$$
\mu \sigma \mu^{-1}=\tau, \quad \mu \tau \mu^{-1}=\rho \sigma
$$

or

$$
\mu \sigma \mu^{-1}=\rho \tau, \quad \mu \tau \mu^{-1}=\sigma .
$$

Then we have further

$$
\mu^{2} \sigma \mu^{-2}=\rho \sigma, \quad \mu^{2} \tau \mu^{-2}=\rho \tau
$$

and

$$
\mu^{3} \sigma \mu^{-3}=\rho \tau, \quad \mu^{3} \tau \mu^{-3}=\sigma
$$

or

$$
\mu^{3} \sigma \mu^{-3}=\tau, \quad \mu^{3} \tau \mu^{-3}=\rho \sigma
$$

Therefore, the subgroups given in the first row are conjugate under $G$. On the other hand, if $C_{G}(\sigma) \neq C_{G}(\tau)$, then there exist $\mu \in C_{G}(\sigma)-C_{G}(\tau)$ and $\nu \in C_{G}(\tau)-C_{G}(\sigma)$. Using these $\mu, \nu$, we can show in the same way as in the first case that the four subgroups are conjugate under $G$. Thus, in both cases, we conclude that the four subgroups are conjugate under $G$.

Since $H \cap E=\langle\sigma, \tau\rangle$, we have

$$
\mathbb{Z} G / H \cong[\mathbb{Z} E /\langle\sigma, \tau\rangle \oplus \mathbb{Z} E /\langle\rho \sigma, \tau\rangle \oplus \mathbb{Z} E /\langle\sigma, \rho \tau\rangle \oplus \mathbb{Z} E /\langle\rho \sigma, \sigma \tau\rangle]^{(t)}
$$


for some $t \geqq 1$ as $E$-lattices. Let

$$
\varepsilon: \mathbb{Z} E /\langle\sigma, \tau\rangle \oplus \mathbb{Z} E /\langle\rho \sigma, \tau\rangle \oplus \mathbb{Z} E /\langle\sigma, \rho \tau\rangle \oplus \mathbb{Z} E /\langle\rho \sigma, \sigma \tau\rangle \rightarrow \mathbb{Z}
$$

be the multiaugmentation map, and set $J=[\operatorname{Ker} \varepsilon]^{\circ}$. Then it follows from Proposition 1.3 that

$$
J_{G / H} \cong J \oplus[\mathbb{Z} E /\langle\sigma, \tau\rangle \oplus \mathbb{Z} E /\langle\rho \sigma, \tau\rangle \oplus \mathbb{Z} E /\langle\sigma, \rho \tau\rangle \oplus \mathbb{Z} E /\langle\rho \sigma, \sigma \tau\rangle]^{(t-1)} .
$$

Since $J$ is not quasi-invertible over $E$ by [E, Theorem 2(1)], this implies that $J_{G / H}$ is not quasi-invertible over $G$. This completes the proof of this step, and so the proof of Theorem 2.1.

Finally, we show the following lemma, which has been used in Step 4.

Lemma 2.2. Let $G=\left\langle\sigma, \tau \mid \sigma^{4}=\tau^{2}=1, \sigma \tau=\tau \sigma\right\rangle$ be the direct product of the cyclic groups $\langle\sigma\rangle$ and $\langle\tau\rangle$. Let

$$
\varepsilon=\left(\varepsilon_{1}, \varepsilon_{2}\right): U=\mathbb{Z} G /\langle\tau\rangle \oplus \mathbb{Z} G /\left\langle\tau \sigma^{2}\right\rangle \rightarrow \mathbb{Z}
$$

be the multiaugmentation map, set $I=\operatorname{Ker} \varepsilon$, and set $J=I^{\circ}$. Then $J$ is not quasi-invertible.

Proof. We construct a concrete coflasque resolution of $I$. The subgroups of $G$ are as follows:

$$
\begin{array}{ll}
\text { order } 1 & \{1\} \\
\text { order } 2 & H_{0}=\left\langle\sigma^{2}\right\rangle, H_{1}=\langle\tau\rangle, H_{2}=\left\langle\tau \sigma^{2}\right\rangle \\
\text { order } 4 & N_{0}=\left\langle\sigma^{2}, \tau\right\rangle, N_{1}=\langle\sigma\rangle, N_{2}=\langle\sigma \tau\rangle \\
\text { order } 8 & G
\end{array}
$$

Under this notation, we have $U=\mathbb{Z} G / H_{1} \oplus \mathbb{Z} G / H_{2}$. Both $\mathbb{Z} G / H_{1}$ and $\mathbb{Z} G / H_{2}$ have $\left\{1, \sigma, \sigma^{2}, \sigma^{3}\right\}$ as representatives of the cosets, so $I$ can be expressed as follows:

$$
I=\mathbb{Z}\langle\sigma\rangle(1,-1)+\mathbb{Z}\langle\sigma\rangle(0, \sigma-1)=\mathbb{Z}\langle\sigma\rangle(1,-1)+\mathbb{Z}\langle\sigma\rangle(\sigma-1,0) .
$$

Here, note that $\tau(1,-1)=(1,-1)-\left(0, \sigma^{2}-1\right)$. Then we have

$$
\begin{aligned}
& I^{H_{1}}=\mathbb{Z}\langle\sigma\rangle\left(1+\sigma^{2}\right)(1,-1)+\mathbb{Z}\langle\sigma\rangle(\sigma-1,0), \\
& I^{H_{2}}=\mathbb{Z}\langle\sigma\rangle\left(1+\sigma^{2}\right)(1,-1)+\mathbb{Z}\langle\sigma\rangle(0, \sigma-1), \\
& I^{H_{0}}=I^{N_{0}}=\mathbb{Z}\langle\sigma\rangle\left(1+\sigma^{2}\right)(1,-1)+\mathbb{Z}\left(1+\sigma^{2}\right)(\sigma-1,0) \\
& \quad=\mathbb{Z}\langle\sigma\rangle\left(1+\sigma^{2}\right)(1,-1)+\mathbb{Z}\left(1+\sigma^{2}\right)(0, \sigma-1),
\end{aligned}
$$




$$
I^{G}=I^{N_{1}}=I^{N_{2}}=\mathbb{Z}\left(1+\sigma+\sigma^{2}+\sigma^{3}\right)(1,-1) \cong \mathbb{Z} .
$$

Define now the $G$-homomorphisms

$$
\begin{aligned}
\delta_{0}: \mathbb{Z} G \rightarrow I & \text { by } \mapsto(1,-1), \\
\delta_{1}: \mathbb{Z} G / H_{1} \rightarrow I & \text { by } 1 \mapsto(\sigma-1,0), \\
\delta_{2}: \mathbb{Z} G / H_{2} \rightarrow I & \text { by } 1 \mapsto(0, \sigma-1), \\
\delta_{3}: \mathbb{Z} G / N_{0} \rightarrow I & \text { by } 1 \mapsto\left(1+\sigma^{2}\right)(1,-1) .
\end{aligned}
$$

Set $V=\mathbb{Z} G \oplus \mathbb{Z} G / H_{1} \oplus \mathbb{Z} G / H_{2} \oplus \mathbb{Z} G / N_{0}$, let

$$
\delta=\left(\delta_{0}, \delta_{1}, \delta_{2}, \delta_{3}\right): V \rightarrow I,
$$

and let $W=\operatorname{Ker} \delta$. Then it is easy to see that $H^{1}(H, W)=0$ for every subgroup $H$ of $G$. This shows that $W$ is coflasque.

We denote by $X^{*}$ the completion of a lattice $X$ at 2 . Then, $\mathbb{Z}^{*} G$ is a local ring, and, for any subgroup $H$ of $G, \mathbb{Z}^{*} G / H$ is indecomposable.

Suppose now that $J$ is quasi-invertible. Then $W$ is invertible by Proposition 1.5(3). Since the Krull-Schmidt theorem holds for permutation lattices over $\mathbb{Z}^{*} G$, the completion $W^{*}$ of $W$ at 2 must be a permutation.

From the exact sequences

$$
\begin{gathered}
0 \rightarrow I \rightarrow U \rightarrow \mathbb{Z} \rightarrow 0, \\
0 \rightarrow W \rightarrow V \rightarrow I \rightarrow 0,
\end{gathered}
$$

we get the list of the $\mathbb{Z}$-rank for these $G$-lattices as follows:

\begin{tabular}{ccccc}
\multicolumn{5}{c}{$H \operatorname{rank}_{\mathbb{Z}} U^{H} \operatorname{rank}_{\mathbb{Z}} I^{H} \operatorname{rank}_{\mathbb{Z}} V^{H} \operatorname{rank}_{\mathbb{Z}} W^{H}$} \\
\hline$\{1\}$ & 8 & 7 & 18 & 11 \\
$H_{0}$ & 4 & 3 & 10 & 7 \\
$H_{1}$ & 6 & 5 & 12 & 7 \\
$H_{2}$ & 6 & 5 & 12 & 7 \\
$N_{0}$ & 4 & 3 & 8 & 5 \\
$N_{1}$ & 2 & 1 & 5 & 4 \\
$N_{2}$ & 2 & 1 & 5 & 4 \\
$G$ & 2 & 1 & 4 & 3
\end{tabular}

From this list, we can deduce that $W^{*} \cong \mathbb{Z}^{*} G \oplus \mathbb{Z}^{*} G / N_{0} \oplus \mathbb{Z}^{*}$. Now, we have the exact sequence

$$
0 \rightarrow W^{*} \rightarrow V^{*} \rightarrow I^{*} \rightarrow 0
$$


with $V^{*} \cong \mathbb{Z}^{*} G \oplus \mathbb{Z}^{*} G / H_{1} \oplus \mathbb{Z}^{*} G / H_{2} \oplus \mathbb{Z}^{*} G / N_{0}$. Setting $V^{\prime}=\mathbb{Z}^{*} G / H_{1} \oplus$ $\mathbb{Z}^{*} G / H_{2} \oplus \mathbb{Z}^{*} G / N_{0}$ and $W^{\prime}=\mathbb{Z}^{*} G / N_{0} \oplus \mathbb{Z}^{*}$, and forming the pushout of

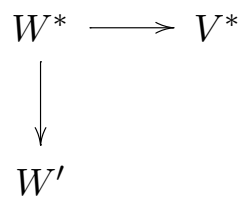

we obtain the exact sequence

$$
0 \rightarrow W^{\prime} \rightarrow V^{\prime} \rightarrow I^{*} \rightarrow 0
$$

which is obviously a contradiction, because the image of $V^{\prime}$ in $I^{*}$ cannot contain the element $(1,-1)$. This concludes that $J$ is not quasi-invertible.

REMARK 2.3. In [CS1, (d3)], it was shown that the torus $R_{K / k}^{(1)}\left(\mathbb{G}_{m}\right)$ is not rational over $k$ in the case where $\operatorname{Gal}(L / k)$ is the dihedral group $D_{4}$ of order 8 and $\operatorname{Gal}(L / K)$ is the subgroup of order 2 .

\section{$\S 3 . \quad$ Metacyclic groups}

The main result of this section is the following.

THEOREM 3.1. Let $K / k$ be a finite non-Galois, separable field extension, and let $L / k$ be the Galois closure of $K / k$. Let $G=\operatorname{Gal}(L / k)$, and let $H=$ $\operatorname{Gal}(L / K) \subseteq G$. Assume that $G$ is metacyclic. Then the following conditions are equivalent.

(1) The norm one torus $R_{K / k}^{(1)}\left(\mathbb{G}_{m}\right)$ defined by $K / k$ is stably rational over $k$.

(2) $G$ is the dihedral group $D_{n}$ of order $2 n$ with $n$ odd $(n \geqq 3)$ or the direct product of the cyclic group $C_{m}$ of order $m$ and the dihedral group $D_{n}$ of order $2 n$, where $m, n$ are odd, $m, n \geqq 3,(m, n)=1$, and $H \subseteq D_{n}$ is of order 2 .

Note that Theorem 3.1(2) is equivalent to the following.

$\left(2^{\prime}\right) H=C_{2}$ is the cyclic group of order 2 , and $G$ is isomorphic to a semidirect product $C_{r} \rtimes H, r \geqq 3$ odd, where $H$ acts nontrivially on the cyclic group $C_{r}$ of order $r$.

Let $G$ be a nonabelian metacyclic group. Then $G$ is expressible as the semidirect product of the cyclic normal subgroup $N_{0}=C_{l}$ of order $l$ by 
the cyclic subgroup $H_{0}=C_{f}$ of order $f$, all Sylow subgroups of which are nonnormal in $G$, where $l \geqq 3$ odd, $f \geqq 2$, and $(f, l)=1$. We define

$$
i(G)=\left|\operatorname{Im}\left(H_{0} \rightarrow \operatorname{Aut} N_{0}\right)\right| .
$$

Theorem 3.1 is only a restatement of the following (see [EM2, (2.3) and p. $\left.\left.92,\left(1^{\prime}\right)\right]\right)$.

TheOREM 3.2. Let $G$ be a nonabelian metacyclic group, and let $H$ be a nonnormal subgroup of $G$ which contains no normal subgroup of $G$ except $\{1\}$. Then the following conditions are equivalent:

(1) $i(G)=2$;

(2) $J_{G / H}$ is a quasi-permutation G-lattice;

(3) $\left[J_{G / H}\right]^{(t)}$ is a quasi-permutation $G$-lattice for some $t \geqq 1$.

REMARK 3.3. The partial results of Theorems 3.1 and 3.2 were obtained in $[\mathrm{CS} 1,(\mathrm{R} 4)$ and $(\mathrm{d} 1)]$ and $[\mathrm{F},(2.3)]$. It is given without proof in [CS1, $(\mathrm{d} 1)]$ that, for the case of $\operatorname{Gal}(L / k)=D_{n}$ with $n$ odd, the torus $R_{K / k}^{(1)}\left(\mathbb{G}_{m}\right)$ is rational over $k$.

Now we will prove Theorem 3.2. In Theorem 3.2 the implication $(2) \Rightarrow$ (3) is obvious, and so it suffices to prove the implications $(1) \Rightarrow(2)$ and $(3) \Rightarrow(1)$.

The proof of $(3) \Rightarrow(1)$. Assume that $i(G) \geqq 3$.

Case 1 . Suppose that $|H| \geqq 3$. Then there exist a subgroup $H^{\prime}$ of $H$ with $\left|H^{\prime}\right|=4$ or $q$ an odd prime and a subgroup $N^{\prime}$ of $N_{0}$ with $\left|N^{\prime}\right|=p$ an odd prime such that $H^{\prime}$ acts faithfully on $N^{\prime}$ by conjugation. Set $G^{\prime}=N^{\prime} H^{\prime}$, and regard $\mathbb{Z} G / H^{\prime}$ as a $G^{\prime}$-lattice. Then we have $\mathbb{Z} G / H \cong \mathbb{Z} G^{\prime} / H^{\prime} \oplus S^{\prime}$ as $G^{\prime}$-lattices, where $S^{\prime}=0$ or $S^{\prime}=\bigoplus_{i=1}^{d} \mathbb{Z} G^{\prime} / H_{i}^{\prime}, d \geqq 1$, for subgroups $H_{i}^{\prime} \subseteq$ $H^{\prime}$, and so, by Proposition $1.3, J_{G / H} \cong J_{G^{\prime} / H^{\prime}} \oplus S^{\prime}$. Therefore, it suffices to show that $\left[J_{G^{\prime} / H^{\prime}}\right]^{(t)}$ is not a quasi-permutation over $G^{\prime}$ for any $t \geqq 1$.

Suppose that $\left[J_{G^{\prime} / H^{\prime}}\right]^{(t)}$ is a quasi-permutation for some $t \geqq 1$. We have an exact sequence

$$
0 \rightarrow I_{G^{\prime} / H^{\prime}} \rightarrow \mathbb{Z} G^{\prime} / H^{\prime} \rightarrow \mathbb{Z} \rightarrow 0
$$

Let $\sigma^{\prime}$ be a generator of $N^{\prime}$. Then $I_{G^{\prime}} / H^{\prime}$ is generated by $\sigma^{\prime}-1$. Therefore, defining the map $\phi: \mathbb{Z} G^{\prime} \rightarrow I_{G^{\prime}} / H^{\prime}$ by $\phi(1)=\sigma^{\prime}-1$ and setting $B^{\prime}=\operatorname{Ker} \phi$, we have an exact sequence

$$
0 \rightarrow B^{\prime} \rightarrow \mathbb{Z} G^{\prime} \rightarrow I_{G^{\prime} / H^{\prime}} \rightarrow 0
$$


It is easy to see that $B^{\prime}$ is coflasque, and so, by Proposition $1.5, B^{\prime}$ is invertible. By assumption, we have $\left[B^{\prime}\right]^{(t)} \oplus U^{\prime} \cong V^{\prime}$ for some permutation $G^{\prime}$-lattices $U^{\prime}$ and $V^{\prime}$, and so $\left[B^{\prime}\right]^{(t)} \oplus U^{\prime} \cong\left[\left[B^{\prime}\right]^{\circ}\right]^{(t)} \oplus U^{\prime}$. From this it follows that $H^{i}\left(G^{\prime}, B^{\prime}\right) \cong H^{i}\left(G^{\prime},\left[B^{\prime}\right]^{\circ}\right)$ for any $i$. Computing the two-dimensional cohomology groups, we obtain

$$
\begin{gathered}
H^{2}\left(G^{\prime}, B^{\prime}\right) \cong H^{1}\left(G^{\prime}, I_{G^{\prime} / H^{\prime}}^{\prime}\right) \cong \mathbb{Z} / p \mathbb{Z} \\
H^{2}\left(G^{\prime},\left[B^{\prime}\right]^{\circ}\right) \cong H^{3}\left(G^{\prime}, J_{G^{\prime} / H^{\prime}}\right) \cong H^{4}\left(G^{\prime}, \mathbb{Z}\right)_{p} \cong H^{4}\left(N^{\prime}, \mathbb{Z}\right)^{H^{\prime}} \cong[\mathbb{Z} / p \mathbb{Z}]^{H^{\prime}},
\end{gathered}
$$

where the $p$-part of a finite abelian group $A$ is denoted by $A_{p}$. This implies that $H^{\prime}$ acts trivially on $H^{4}\left(N^{\prime}, \mathbb{Z}\right)$, but, according to $[\mathrm{Br}$, p. 159 , Example 6], this is not the case because of $\left|H^{\prime}\right|=4$ or $q$.

Case 2. Suppose that $|H|=2$.

If there exists an odd prime $q \mid i(G)$, then there exist a subgroup $H^{\prime}$ of $H_{0}$ with $\left|H^{\prime}\right|=q$ and a subgroup $N^{\prime}$ of $N_{0}$ with $\left|N^{\prime}\right|=p$ an odd prime such that $H^{\prime}$ acts nontrivially on $N^{\prime}$ by conjugation. Setting $G^{\prime}=N^{\prime} H^{\prime}$, and regarding $\mathbb{Z} G / H$ as a $G^{\prime}$-lattice, we have $\mathbb{Z} G / H \cong\left[\mathbb{Z} G^{\prime}\right]^{(s)}$ for some $s \geqq 1$, as $G^{\prime}$ lattices, and therefore, by Corollary 1.4, $J_{G / H} \cong J_{G^{\prime}} \oplus\left[\mathbb{Z} G^{\prime}\right]^{(s-1)}$. According to $[\mathrm{EM} 2,(2.3)],\left[J_{G^{\prime}}\right]^{(t)}$ is not a quasi-permutation over $G^{\prime}$ for any $t \geqq 1$. Thus, we conclude that $\left[J_{G / H}\right]^{(t)}$ is not a quasi-permutation over $G$ for any $t \geqq 1$.

If $i(G)(\geqq 3)$ is a power of 2 , then there exist a subgroup $H^{\prime}$ of $H_{0}$ with $\left|H^{\prime}\right|=4$ containing $H$ and a subgroup $N^{\prime}$ of $N_{0}$ with $\left|N^{\prime}\right|=p$ an odd prime such that $H^{\prime}$ acts faithfully on $N^{\prime}$ by conjugation. Setting $G^{\prime}=N^{\prime} H^{\prime}$, and regarding $\mathbb{Z} G / H$ as a $G^{\prime}$-lattice, we have $\mathbb{Z} G / H \cong \mathbb{Z} G^{\prime} / H \oplus S^{\prime}$ as $G^{\prime}$-lattices, where $S^{\prime}=0$ or $S^{\prime}=\bigoplus_{i=1}^{d} \mathbb{Z} G^{\prime} / H_{i}, d \geqq 1$, for subgroups $H_{i} \subseteq H$, and so, by Proposition $1.3, J_{G / H} \cong J_{G^{\prime} / H} \oplus S^{\prime}$. Therefore, it suffices to show that $\left[J_{G^{\prime} / H}\right]^{(t)}$ is not a quasi-permutation over $G^{\prime}$. Suppose that $\left[J_{G^{\prime} / H}\right]^{(t)}$ is a quasi-permutation for some $t \geqq 1$. We have an exact sequence

$$
0 \rightarrow I_{G^{\prime} / H} \rightarrow \mathbb{Z} G^{\prime} / H \rightarrow \mathbb{Z} \rightarrow 0
$$

Let $N^{\prime}=\langle\sigma\rangle$, and let $H^{\prime}=\langle\tau\rangle$. Then we have $I_{G^{\prime} / H}=(\sigma-1, \tau-1)$. Noticing that $\left[I_{G^{\prime} / H}\right]^{N^{\prime}}=\mathbb{Z}\left(\sum_{i=0}^{p-1} \sigma^{i}\right)(\tau-1)$, we can construct the following coflasque resolution of $I_{G^{\prime} / H}$ :

$$
0 \rightarrow B^{\prime} \rightarrow \mathbb{Z} G^{\prime} \oplus \bigoplus_{i=1}^{s} \mathbb{Z} G^{\prime} / H_{i}^{\prime} \rightarrow I_{G^{\prime}} / H \rightarrow 0
$$


where each $H_{i}^{\prime}$ is a subgroup of $H^{\prime}$. Then $B^{\prime}$ is invertible by [EM2, (1.5)]. Since $\left[J_{G^{\prime} / H}\right]^{(t)}$ is a quasi-permutation, $\left[B^{\prime}\right]^{(t)}$ is a quasi-permutation by Proposition 1.5, and so we have $\left[B^{\prime}\right]^{(t)} \oplus U^{\prime} \cong V^{\prime}$ for some permutation $G^{\prime}$-lattices $U^{\prime}$ and $V^{\prime}$. From this it follows that $H^{i}\left(G^{\prime}, B^{\prime}\right) \cong H^{i}\left(G^{\prime},\left[B^{\prime}\right]^{\circ}\right)$ for any $i$. Computing the cohomology groups $H^{2}\left(G^{\prime}, B^{\prime}\right)$ and $H^{2}\left(G^{\prime},\left[B^{\prime}\right]^{\circ}\right)$ along the same lines as in Case 1 , and considering only the $p$-parts of the cohomology groups, we finally see that

$$
\mathbb{Z} / p \mathbb{Z} \cong H^{4}\left(N^{\prime}, \mathbb{Z}\right)^{H^{\prime}} \cong[\mathbb{Z} / p \mathbb{Z}]^{H^{\prime}}
$$

which is a contradiction. This completes the proof $(3) \Rightarrow(1)$.

The proof of $(1) \Rightarrow(2)$. Assume that $i(G)=2$.

Under this assumption, the subgroups of $H$ of $G$ as in the theorem are of order 2. Therefore, $G$ and $H$ are expressible as follows:

$$
G=\left\langle\mu, \nu, \tau \mid \mu^{m}=\nu^{n}=\tau^{2}=1, \mu \nu=\nu \mu, \mu \tau=\tau \mu, \tau \nu \tau^{-1}=\nu^{-1}\right\rangle
$$

and $H=\langle\tau\rangle$, where $m, n$ are odd, $m \geqq 1, n \geqq 3$, and $(m, n)=1$, that is, that $G=\langle\mu\rangle \times\langle\nu, \tau\rangle$, the direct product of the cyclic group $C_{m}$ of order $m$ and the dihedral group $D_{n}$ of order $2 n$.

Now we will prove that $J_{G / H}$ is a quasi-permutation, by induction on the number of prime divisors of $n$. We denote by $\Phi_{a}(X)$ the ath cyclotomic polynomial and by $\zeta_{a}$ the primitive $a$ th root of unity.

Set $\sigma=\mu \nu$, and set $l=m n$. Let $p$ be a prime divisor of $n$. Let $n=p^{c} n^{\prime}$, $p \nmid n^{\prime}$, and let $l^{\prime}=l / p^{c}$. Further, let $\Psi(X)=\prod_{r \mid l^{\prime}} \Phi_{p^{c} r}(X)$ and $\Psi_{0}(X)=$ $\Psi(X) / \Phi_{p^{c}}(X)$, and let $\Gamma=\mathbb{Z} G /(\Psi(\sigma)), \Gamma_{0}=\mathbb{Z} G /\left(\Psi_{0}(\sigma)\right)$, and $\Gamma_{1}=\mathbb{Z} G /$ $\left(\Phi_{p^{c}}(\sigma)\right)$. Then there is an exact sequence of $G$-lattices

$$
0 \rightarrow \Gamma_{1} \rightarrow \Gamma \rightarrow \Gamma_{0} \rightarrow 0
$$

From now on, the tensor products $\otimes$ mean those over $\mathbb{Z} G$ for brevity. As is easily seen, $\Gamma_{1} \otimes I_{G / H}, \Gamma \otimes I_{G / H}$, and $\Gamma_{0} \otimes I_{G / H}$ are torsion free, and hence the following sequence is exact:

$$
0 \rightarrow \Gamma_{1} \otimes I_{G / H} \rightarrow \Gamma \otimes I_{G / H} \rightarrow \Gamma_{0} \otimes I_{G / H} \rightarrow 0 .
$$

From the fact that $\Psi_{0}(1)= \pm 1$, it follows that $\Gamma_{0} \otimes \mathbb{Z}=0$, and so, tensoring $\Gamma_{0}$ with the exact sequence $0 \rightarrow I_{G / H} \rightarrow \mathbb{Z} G / H \rightarrow \mathbb{Z} \rightarrow 0$, we have

$$
\Gamma_{0} \otimes I_{G / H} \cong \Gamma_{0} \otimes \mathbb{Z} G / H
$$


Let $\eta(X)=\left(X^{l}-1\right) / \Psi_{0}(X)=\left(X^{l / p}-1\right) \Phi_{p^{c}}(X)$, and let $\Gamma^{\prime}=\mathbb{Z} G /(\eta(\sigma))$. Then we have the following exact sequence:

$$
0 \rightarrow \Gamma^{\prime} \rightarrow \mathbb{Z} G \rightarrow \Gamma_{0} \rightarrow 0 .
$$

Tensoring $\mathbb{Z} G / H$ with this exact sequence, we obtain the following exact sequence:

$$
0 \rightarrow \Gamma^{\prime} \otimes \mathbb{Z} G / H \rightarrow \mathbb{Z} G / H \rightarrow \Gamma_{0} \otimes \mathbb{Z} G / H \rightarrow 0 .
$$

Using these facts, we can form the following pullback diagram with exact rows and columns:

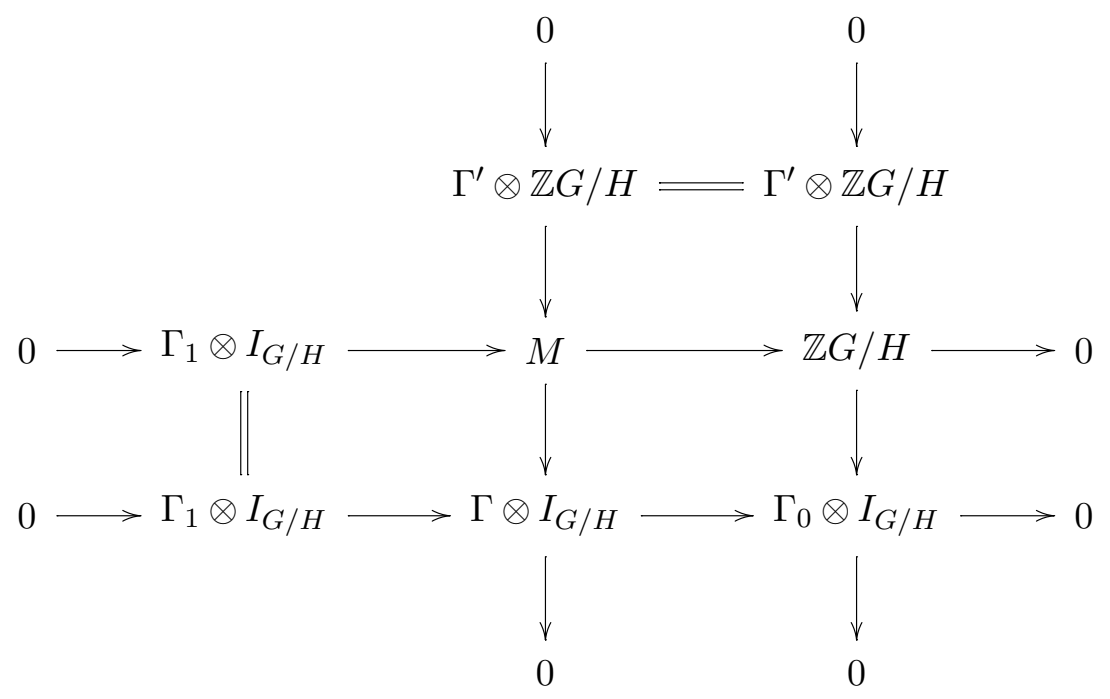

Now, $\Gamma_{1} \cong \mathbb{Z}\left[\zeta_{p^{c}}, \tau\right]$ is the twisted group ring of $H$ over $\mathbb{Z}\left[\zeta_{p^{c}}\right]$, and $\Gamma_{1} \otimes$ $I_{G / H} \cong\left(\zeta_{p^{c}}-1\right) \subseteq \mathbb{Z}\left[\zeta_{p^{c}}\right]$ is an ambiguous ideal of $\mathbb{Z}\left[\zeta_{p^{c}}\right]$. As is easily seen, $\mathbb{Q}\left(\zeta_{p^{c}}\right)$ is tamely ramified over $\mathbb{Q}\left(\zeta_{p^{c}}\right)^{H}=\mathbb{Q}\left(\zeta_{p^{c}}+\zeta_{p^{c}}^{-1}\right)$, and then $\Gamma_{1}$ is a nonmaximal, hereditary order in the full matrix algebra $M_{2}\left(\mathbb{Q}\left(\zeta_{p^{c}}+\zeta_{p^{c}}^{-1}\right)\right)$ of degree 2 over $\mathbb{Q}\left(\zeta_{p^{c}}+\zeta_{p^{c}}^{-1}\right)$. Setting $S=\mathbb{Z}\left[\zeta_{p^{c}}\right]$ and $P=\left(\zeta_{p^{c}}-1\right)$, we have $\Gamma_{1} \cong S \oplus P$ as $\Gamma_{1}$-lattices and $\left(\Gamma_{1}\right)^{\circ} \cong \Gamma_{1}, S^{\circ} \cong S$. Hence, all of $\Gamma_{1},\left(\Gamma_{1}\right)^{\circ}$, $S^{\circ}, S, P, P^{\circ}$ are $\Gamma_{1}$-projective (see $[\mathrm{R}],\left[\mathrm{CR}\right.$, Section 28]). Since $\Gamma_{1}$ is $\mathbb{Z} H$ free, so is $\Gamma_{1} \otimes I_{G / H}$. Therefore, we have

$$
\operatorname{Ext}_{\mathbb{Z} G}^{1}\left(\mathbb{Z} G / H, \Gamma_{1} \otimes I_{G / H}\right) \cong H^{1}\left(H, \Gamma_{1} \otimes I_{G / H}\right)=0 .
$$

Accordingly, the second row of the above diagram is split, and so we obtain the exact sequence

$$
0 \rightarrow \Gamma^{\prime} \otimes \mathbb{Z} G / H \rightarrow\left[\Gamma_{1} \otimes I_{G / H}\right] \oplus \mathbb{Z} G / H \rightarrow \Gamma \otimes I_{G / H} \rightarrow 0 .
$$


We further see that $\left[\Gamma_{1} \otimes I_{G / H}\right]^{\circ}\left(\cong P^{\circ}\right)$ is a quasi-permutation $G /\left\langle\sigma^{p^{c}}\right\rangle$ lattice, because both $\Gamma_{1}$ and $S$ are quasi-permutations. On the other hand, there is an exact sequence

$$
0 \rightarrow \mathbb{Z} G /\left\langle\sigma^{l / p}\right\rangle \rightarrow \Gamma^{\prime} \rightarrow \Gamma_{1} \rightarrow 0 .
$$

Tensoring $\mathbb{Z} G / H$ with this, we obtain an exact sequence

$$
0 \rightarrow \mathbb{Z} G /\left\langle\sigma^{l / p}\right\rangle \otimes \mathbb{Z} G / H \rightarrow \Gamma^{\prime} \otimes \mathbb{Z} G / H \rightarrow \Gamma_{1} \otimes \mathbb{Z} G / H \rightarrow 0
$$

Since $\mathbb{Z} G /\left\langle\sigma^{l / p}\right\rangle \otimes \mathbb{Z} G / H$ is a permutation and $\Gamma_{1} \otimes \mathbb{Z} G / H \cong \mathbb{Z}\left[\zeta_{p^{c}}\right],\left[\Gamma^{\prime} \otimes\right.$ $\mathbb{Z} G / H]^{\circ}$ is also a quasi-permutation. Hence, setting $U=\left[\Gamma_{1} \otimes I_{G / H}\right]^{\circ} \oplus$ $\mathbb{Z} G / H$ and $V=\left[\Gamma^{\prime} \otimes \mathbb{Z} G / H\right]^{\circ}$, we have an exact sequence

$$
0 \rightarrow\left[\Gamma \otimes I_{G / H}\right]^{\circ} \rightarrow U \rightarrow V \rightarrow 0
$$

where both $U$ and $V$ are quasi-permutations.

Let $\bar{G}=G /\left\langle\sigma^{m n^{\prime} p^{c-1}}\right\rangle$. Note that $\mathbb{Z} \bar{G}=\mathbb{Z} G /\left\langle\sigma^{m n^{\prime} p^{c-1}}\right\rangle \cong \Psi(\sigma) \mathbb{Z} G$ and that $\Psi(1)=p$. Then we can form the following commutative diagram with exact rows and columns:

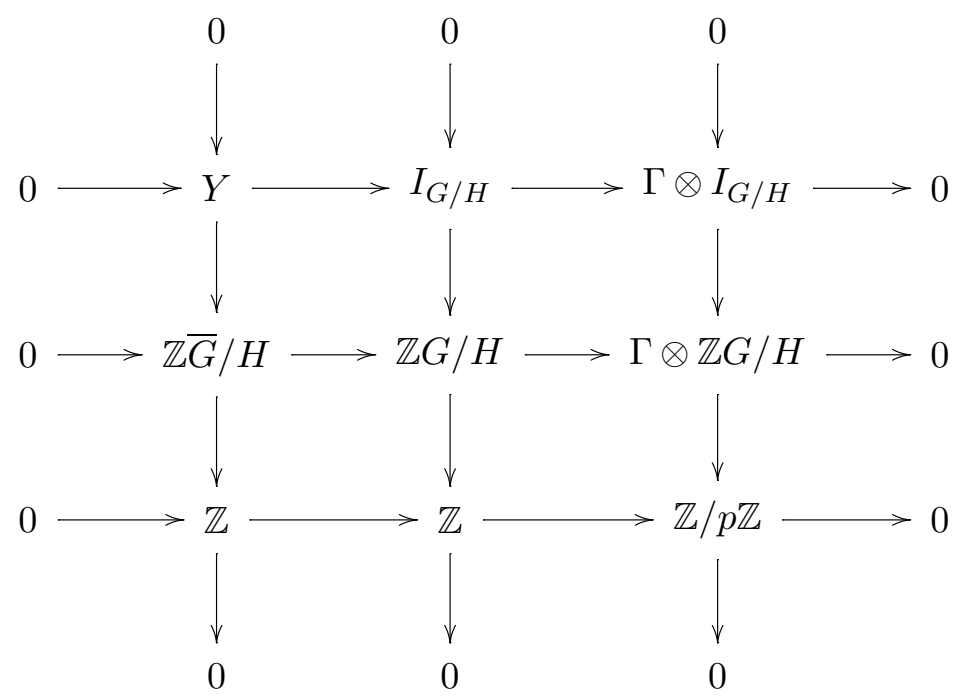

It is easy to see that $Y \cong I_{\bar{G} / H}$, and so we have an exact sequence

$$
0 \rightarrow\left[\Gamma \otimes I_{G / H}\right]^{\circ} \rightarrow J_{G / H} \rightarrow J_{\bar{G} / H} \rightarrow 0
$$


Finally, we show that, for any subgroup $G^{\prime}$ of $G$,

$$
H^{0}\left(G^{\prime},\left[\Gamma \otimes I_{G / H}\right]^{\circ}\right)=H^{0}\left(G^{\prime}, \Gamma \otimes I_{G / H}\right)=0 .
$$

In order to show (iii), we first prove that

$$
H^{0}\left(G^{\prime}, \Gamma \otimes \mathbb{Z} G / H\right)=0 \text { for any } G^{\prime} \subseteq G .
$$

By definition, $\Psi(X)=\Phi_{p}\left(X^{m n^{\prime} p^{c-1}}\right)$, and so $\Gamma \otimes \mathbb{Z} G / H=\mathbb{Z}[\sigma] /(\Psi(\sigma))=$ $\mathbb{Z}[\sigma] /\left(\Phi_{p}\left(\sigma^{m n^{\prime} p^{c-1}}\right)\right) \cong \mathbb{Z}\left[\zeta_{p}\right]+\mathbb{Z}\left[\zeta_{p}\right] \sigma+\mathbb{Z}\left[\zeta_{p}\right] \sigma^{2}+\cdots+\mathbb{Z}\left[\zeta_{p}\right] \sigma^{m n^{\prime} p^{c-1}(p-1)-1}$, where $\sigma^{m n^{\prime} p^{c-1}}=\zeta_{p}$. From this it follows that $[\Gamma \otimes \mathbb{Z} G / H]^{N_{0}}=0$, where $N_{0}=\left\langle\sigma^{m n^{\prime} p^{c-1}}\right\rangle$.

Assume first that $N_{0} \subseteq G^{\prime}$. Then $[\Gamma \otimes \mathbb{Z} G / H]^{G^{\prime}}=0$, so that $H^{0}\left(G^{\prime}, \Gamma \otimes\right.$ $\mathbb{Z} G / H)=0$. Next assume that $G^{\prime} \subseteq N$. From the exact sequence

$$
0 \rightarrow \mathbb{Z} G /\left\langle\sigma^{m n^{\prime} p^{c-1}}, \tau\right\rangle \rightarrow \mathbb{Z} G / H \rightarrow \Gamma \otimes \mathbb{Z} G / H \rightarrow 0,
$$

we obtain the following exact sequence:

$$
\rightarrow H^{0}\left(G^{\prime}, \mathbb{Z} G / H\right) \rightarrow H^{0}\left(G^{\prime}, \Gamma \otimes \mathbb{Z} G / H\right) \rightarrow H^{1}\left(G^{\prime}, \mathbb{Z} G /\left\langle\sigma^{m n^{\prime} p^{c-1}}, \tau\right\rangle\right) \rightarrow
$$

Since $\mathbb{Z} G / H \cong \mathbb{Z} N$ as $N$-lattices, $H^{0}\left(G^{\prime}, \mathbb{Z} G / H\right)=0$, and since $\mathbb{Z} G /$ $\left\langle\sigma^{m n^{\prime} p^{c-1}}, \tau\right\rangle$ is a permutation, $H^{1}\left(G^{\prime}, \mathbb{Z} G /\left\langle\sigma^{m n^{\prime} p^{c-1}}, \tau\right\rangle\right)=0$. Thus, we have $H^{0}\left(G^{\prime}, \Gamma \otimes \mathbb{Z} G / H\right)=0$. Further, assume that $G^{\prime}=H=\langle\tau\rangle$ (or one of its conjugates $)$. Then, we have $N_{G^{\prime}}\left(-\left(\zeta_{p}+\zeta_{p}^{2}+\cdots+\zeta_{p}^{(p-1) / 2}\right) u\right)=u$ for any $u \in$ $[\Gamma \otimes \mathbb{Z} G / H]^{G^{\prime}}$, which implies that $H^{0}\left(G^{\prime}, \Gamma \otimes \mathbb{Z} G / H\right)=0$. In the other cases, we may assume that $G^{\prime}=\left\langle\sigma^{m^{\prime} n^{\prime \prime} p^{c}}, \tau\right\rangle$, where $m^{\prime}\left|m, n^{\prime \prime}\right| n^{\prime}$ and $m^{\prime}<m$ or $n^{\prime \prime}<n$. Set $N^{\prime}=\left\langle\sigma^{m^{\prime} n^{\prime \prime} p^{c}}\right\rangle$. Then we have $[\Gamma \otimes \mathbb{Z} G / H]^{N^{\prime}}=\left(1+\mu^{m^{\prime}}+\cdots+\right.$ $\left.\left(\mu^{m^{\prime}}\right)^{m / m^{\prime}-1}\right)\left(1+\nu^{n^{\prime \prime}}+\cdots+\left(\nu^{n^{\prime \prime}}\right)^{n^{\prime} / n^{\prime \prime}-1}\right) \Gamma \otimes \mathbb{Z} G / H$, and therefore $[\Gamma \otimes$ $\mathbb{Z} G / H]^{G^{\prime}}=\left(1+\mu^{m^{\prime}}+\cdots+\left(\mu^{m^{\prime}}\right)^{m / m^{\prime}-1}\right)\left(1+\nu^{n^{\prime \prime}}+\cdots+\left(\nu^{n^{\prime \prime}}\right)^{n^{\prime} / n^{\prime \prime}-1}\right)[\Gamma \otimes$ $\mathbb{Z} G / H]^{H}=N_{G^{\prime}}(\Gamma \otimes \mathbb{Z} G / H)$, which implies that $H^{0}\left(G^{\prime}, \Gamma \otimes \mathbb{Z} G / H\right)=0$. This concludes the proof of (iii').

From (iii') and the exact sequence

$$
0 \rightarrow \Gamma \otimes I_{G / H} \rightarrow \Gamma \otimes \mathbb{Z} G / H \rightarrow \mathbb{Z} / p \mathbb{Z} \rightarrow 0
$$

we obtain an exact sequence

$$
H^{-1}\left(G^{\prime}, \Gamma \otimes \mathbb{Z} G / H\right) \stackrel{\theta}{\longrightarrow} H^{-1}\left(G^{\prime}, \mathbb{Z} / p \mathbb{Z}\right) \rightarrow H^{0}\left(G^{\prime}, \Gamma \otimes I_{G / H}\right) \rightarrow 0
$$


for any $G^{\prime} \subseteq G$. The above map $\theta: H^{-1}\left(G^{\prime}, \Gamma \otimes \mathbb{Z} G / H\right) \rightarrow H^{-1}\left(G^{\prime}, \mathbb{Z} / p \mathbb{Z}\right)$ is surjective. In fact, we have $H^{-1}\left(G^{\prime}, \mathbb{Z} / p \mathbb{Z}\right)=0, \mathbb{Z} / p \mathbb{Z}$ when $p \nmid\left|G^{\prime}\right|, p|| G^{\prime} \mid$, respectively. For the case where $p|| G^{\prime} \mid, N_{0}=\left\langle\sigma^{m n^{\prime} p^{c-1}}\right\rangle \subseteq G^{\prime}$ and $N_{N_{0}}(\Gamma \otimes$ $\mathbb{Z} G / H)=\left(1+\zeta_{p}+\zeta_{p}^{2}+\cdots+\zeta_{p}^{p-1}\right) \Gamma \otimes \mathbb{Z} G / H=0$, and therefore Ker $N_{G^{\prime}}=$ $\operatorname{Ker} N_{N_{0}}=\Gamma \otimes \mathbb{Z} G / H$. Thus, $\theta$ is surjective; that is, $H^{0}\left(G^{\prime}, \Gamma \otimes I_{G / H}\right)=0$, which completes the proof of (iii).

By (i), (iii), and [EM2, (2.2)], $\left[\Gamma \otimes I_{G / H}\right]^{\circ}$ is a quasi-permutation. Further, by (ii) and $[\mathrm{EM} 2,(2.2)], J_{G / H}$ is a quasi-permutation if and only if $J_{\bar{G} / H}$ is so. Note that, for the case where $n=p, \bar{G}$ is cyclic of order $2 m$, and therefore $J_{\bar{G} / H}$ is a quasi-permutation. Hence, by induction, we can show that $J_{G / H}$ is a quasi-permutation. This completes the proof of the implication $(1) \Rightarrow(2)$.

REMARK 3.4. The above proof of $(3) \Rightarrow(1)$ was done in the same way as in [CS1, (R4)]. The proof of $(1) \Rightarrow(2)$ was done by making some modifications on that in $[\mathrm{EM} 2,(2.3)]$.

\section{§4. Symmetric groups and alternating groups}

In this section, we consider the problem for $S_{n}$ (resp., $A_{n}$ ), the symmetric (resp., alternating) group on $n$ letters. We also assume that the subgroup $S_{n-1}$ (resp., $A_{n-1}$ ) of $S_{n}$ (resp., $A_{n}$ ) is the stabilizer of one of the letters in $S_{n}$ (resp., $\left.A_{n}\right)$.

Let $K / k$ be a non-Galois separable field extension of degree $n$, and let $L / k$ be the Galois closure of $K / k$. Let $T_{n}=R_{K / k}^{(1)}\left(\mathbb{G}_{m}\right)$ be the norm one torus defined by $K / k$.

We give first the following.

Theorem 4.1. Assume that $\operatorname{Gal}(L / k)=S_{n}, n \geqq 2$, and that $\operatorname{Gal}(L / K)=$ $S_{n-1}$. Then,

(1) $T_{n}$ is retract rational over $k$ if and only if $n$ is a prime;

(2) $T_{n}$ is (stably) rational over $k$ if and only if $n=2,3$.

REMARK 4.2. The "if" part of Theorem 4.1(1) was first proved in [CS2]. It is well known that, for $n=2,3, T_{n}$ is rational over $k$. The "only if" part of Theorem 4.1(2) was proved in [1B] for the case where $n$ is a prime, and in $[\mathrm{CK}]$ for the general case. Note that the "only if" parts of Theorem 4.1(1), (2) were proved implicitly in [LL].

Theorem 4.1 can be restated as follows. 
THEOREM 4.3. Let $S_{n}, n \geqq 2$ be the symmetric group on $n$ letters. Then we have that

(1) $J_{S_{n} / S_{n-1}}$ is quasi-invertible over $S_{n}$ if and only if $n$ is a prime;

(2) $J_{S_{n} / S_{n-1}}$ is a quasi-permutation over $S_{n}$ if and only if $n=2,3$.

Proof. The "if" part of (1) is only a corollary to Proposition 1.7 because $S_{n-1}$ is a Hall subgroup of $S_{n}$ if $n$ is a prime. Suppose now that $n$ is not a prime.

First assume that there is an odd prime $p \mid n$, and set $m=n / p \geqq 2$. Let $P$ be the elementary abelian $p$-subgroup of $S_{n}$ generated by $\rho_{1}=\left(\begin{array}{llll}1 & 2 & \cdots & p\end{array}\right)$, $\rho_{2}=(p+1 p+2 \cdots 2 p), \ldots, \rho_{m}=((m-1) p+1(m-1) p+2 \cdots m p)$, and set further $P_{1}=\left\langle\rho_{2}, \rho_{3}, \ldots, \rho_{m}\right\rangle, P_{2}=\left\langle\rho_{1}, \rho_{3}, \ldots, \rho_{m}\right\rangle, \ldots, P_{m}=\left\langle\rho_{1}, \rho_{2}\right.$, $\left.\ldots, \rho_{m-1}\right\rangle$. Regarding $\mathbb{Z} S_{n} / S_{n-1}$ as $P$-lattices, we have

$$
\mathbb{Z} S_{n} / S_{n-1} \cong \mathbb{Z} P / P_{1} \oplus \mathbb{Z} P / P_{2} \oplus \cdots \oplus \mathbb{Z} P / P_{m},
$$

and therefore, by [E, Theorem 2(2)], $J_{S_{n} / S_{n-1}}$ is not quasi-invertible over $P$. This implies that $J_{S_{n} / S_{n-1}}$ is not quasi-invertible over $S_{n}$.

Assume next that $n=2^{h}, h \geqq 2$. Let $P$ be the subgroup of $S_{n}$ gener-

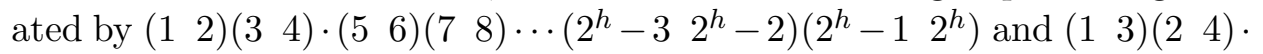
$\left(\begin{array}{ll}5 & 7\end{array}\right)\left(\begin{array}{ll}6 & 8\end{array}\right) \cdots\left(2^{h}-32^{h}-1\right)\left(2^{h}-22^{h}\right)$. Then $P$ is an elementary abelian group of order 4 , and, as is easily seen, $\mathbb{Z} S_{n} / S_{n-1} \cong[\mathbb{Z} P]^{\left(2^{h-2}\right)}$ as $P$-lattices. Since $J_{P}$ is not quasi-invertible by Theorem $1.2(1)$, it follows from Corollary 1.4 that $J_{S_{n} / S_{n-1}}$ is not quasi-invertible over $S_{n}$.

For assertion (2), the "if" part is well known, and so it suffices to prove the "only if" part. However, for $n$ a nonprime, this follows directly from assertion (1). Hence it remains to prove this for $n=p \geqq 5$ a prime. Let $\sigma=\left(\begin{array}{llll}1 & 2 & \cdots & p\end{array}\right)$, and let $\tau$ be a $(p-1)$ cycle on the letters $2,3, \ldots, p$ acting faithfully on $\langle\sigma\rangle$ by conjugation. Set $G^{\prime}=\langle\sigma, \tau\rangle$, and set $H^{\prime}=\langle\tau\rangle$. Then we have $\mathbb{Z} S_{p} / S_{p-1} \cong \mathbb{Z} G^{\prime} / H^{\prime}$, and so $J_{S_{p} / S_{p-1}} \cong J_{G^{\prime} / H^{\prime}}$ as $G^{\prime}$-lattices. Since $p-1 \geqq 4$, it follows from Theorem 3.2 that $J_{G^{\prime} / H^{\prime}}$ is not a quasi-permutation, and so $J_{S_{p} / S_{p-1}}$ is not a quasi-permutation over $S_{p}$. Thus, the proof is complete.

Note that Theorem 4.3(2) can be replaced by the following:

$\left(2^{\prime}\right)\left[J_{S_{n} / S_{n-1}}\right]^{(t)}$ is a quasi-permutation for some $t \geqq 1$ if and only if $n=2,3$.

Next, we give the following.

Theorem 4.4. Assume that $\operatorname{Gal}(L / k)=A_{n}, n \geqq 3$ and that $\operatorname{Gal}(L / K)=$ $A_{n-1}$. Then, 
(1) $T_{n}$ is retract rational over $k$ if and only if $n$ is a prime;

(2) $\left[T_{n}\right]^{(t)}$ is stably rational over $k$ for some $t \geqq 1$ if and only if $n=3,5$.

This can also be reduced to the following.

THEOREM 4.5. Let $A_{n}, n \geqq 3$ be the alternating group on $n$ letters. Then,

(1) $J_{A_{n} / A_{n-1}}$ is quasi-invertible if and only if $n$ is a prime;

(2) $\left[J_{A_{n} / A_{n-1}}\right]^{(t)}$ is a quasi-permutation for some $t \geqq 1$ if and only if $n=$ 3,5 .

Proof. The "if" part of (1) is only a corollary to Proposition 1.7 because $A_{n-1}$ is a Hall subgroup of $A_{n}$ if $n$ is a prime. In the case where $n$ is not a prime, the assertions can be proved by the same way as in Theorem 4.3. Thus, the proof of (1) is complete. In order to show (2), we may assume that $n=p \geqq 3$ is a prime. The $(p-1)$ cycle $\tau$ in the proof of Theorem 4.3(2) is not contained in $A_{p}$. Therefore, we must use $G^{\prime \prime}=\left\langle\sigma, \tau^{2}\right\rangle$ and $H^{\prime \prime}=\left\langle\tau^{2}\right\rangle$ instead of $G^{\prime}$ and $H^{\prime}$, respectively, in the proof of Theorem 4.3. Then, by Theorem 3.2 , we see that $\left[J_{G^{\prime \prime} / H^{\prime \prime}}\right]^{(t)}$ is a quasi-permutation for some $t \geqq 1$ if and only if $p=3,5$. Since $\mathbb{Z} A_{p} / A_{p-1} \cong \mathbb{Z} G^{\prime \prime} / H^{\prime \prime}$ and $J_{A_{p} / A_{p-1}} \cong J_{G^{\prime \prime} / H^{\prime \prime}}$ as $G^{\prime \prime}$-lattices, this also shows that $\left[J_{A_{p} / A_{p-1}}\right]^{(t)}$ is not a quasi-permutation over $A_{p}$ for any $t \geqq 1$ when $p \geqq 7$. On the other hand, $J_{A_{3} / A_{2}}$ is a quasipermutation because $A_{3}$ is cyclic of order 3 . Further, according to [D, (3.3)], $\left[J_{A_{5} / A_{4}}\right]^{(t)}$ is a quasi-permutation for some $t \geqq 1$. This completes the proof of $(2)$.

REMARK 4.6. It is an open problem whether $J_{A_{5} / A_{4}}$ is a quasi-permutation. This is an interesting problem because we do not know any example of the norm one torus defined by non-Galois separable extension $K / k$ which is stably rational over $k$ except those in Theorem 3.1 .

\section{REFERENCES}

[Be] Y. Berkovich, Groups of Prime Power Order, I, de Gruyter, Berlin, 2008.

[Br] K. S. Brown, Cohomology of Groups, Grad. Texts in Math. 87, Springer, New York, 1982.

[CS1] J.-L. Colliot-Thélène and J.-J. Sansuc, La R-équivalence sur les tores, Ann. Sci. Éc. Norm. Supér. (4) 10 (1977), 175-230.

[CS2] , Principal homogeneous spaces under flasque tori: Applications, J. Algebra 106 (1987), 148-205.

[CK] A. Cortella and B. Kunyavskiı̌, Rationality problem for generic tori in simple groups, J. Algebra 225 (2000), 771-793.

[CR] C. W. Curtis and I. Reiner, Methods of Representation Theory, I, John Wiley, New York, 1981. 
[D] A. Dress, The permutation class group of a finite group, J. Pure Appl. Algebra 6 (1975), 1-12.

[E] S. Endo, On the rationality of algebraic tori of norm type, J. Algebra 235 (2001), $27-35$.

[EM1] S. Endo and T. Miyata, Invariants of finite abelian groups, J. Math. Soc. Japan 25 (1973), 7-26.

[EM2] - On a classification of the function fields of algebraic tori, Nagoya Math. J. 56 (1975), 85-104; Correction, Nagoya Math. J. 79 (1980), 187-190.

[F] M. Florence, Non rationality of some norm one tori, preprint, 2006.

[IB] L. Le Bruyn, Generic norm one tori, Nieuw Arch. Wiskd. (5) 13 (1995), 401-407.

[LL] N. Lemire and M. Lorenz, On certain lattices associated with generic division algebras, J. Group Theory 3 (2000), 385-405.

[R] M. Rosen, Representations of twisted group rings, Ph.D. dissertation, Princeton University, Princeton, N. J., 1963.

[S] D. J. Saltman, Retract rational fields and cyclic Galois extensions, Israel J. Math. 47 (1984), 165-215.

[V] V. E. Voskresenskiŭ, Algebraic Groups and Their Birational Invariants, Transl. Math. Monogr. 179, Amer. Math. Soc., Providence, 1998.

Uehara $3-4-9$

Shibuya-ku

Tokyo 151-0064

Japan

wktxj285@yahoo.co.jp 